\title{
Intervenciones de cambio de comportamiento para la reducción del consumo de alcohol durante el embarazo.
}

Respuesta rápida de un tópico valorado críticamente.

Behavior change interventions for reducing alcohol consumption during pregnancy.

Autores:

Luis Ortiz-Muñoz L

ORCID: 0000-0001-6449-2153

Centro Evidencia UC, Pontificia Universidad Católica de Chile.

Satellite Cochrane EPOC-Chile.

Alejandra Parada

ORCID: 0000-0001-9573-2957

Departamento Ciencias de la Salud, carrera de Nutrición y Dietética, Facultad de Medicina, Pontificia Universidad Católica de Chile.

\section{Carolina Fredes}

ORCID: 0000-0002-2786-9078

Departamento Ciencias de la Salud, carrera de Nutrición y Dietética, Facultad de Medicina, Pontificia Universidad Católica de Chile.

\section{Loreto Rojas}

ORCID: 0000-0002-6394-4733

Departamento Ciencias de la Salud, carrera de Nutrición y Dietética, Facultad de Medicina, Pontificia Universidad Católica de Chile.

\section{Giuliano Duarte}

ORCID: 0000-0001-9724-0796

Faculty of Medical Sciences, Midwifery School, Universidad de Santiago de Chile, Santiago, Chile

Josep M. Suelves

ORCID: 0000-0002-7716-6944

Agència de Salut Pública de Catalunya, Barcelona, España

Universitat Oberta de Catalunya - UOC, Barcelona, España

\section{Manuel Armayones Ruiz}

ORCID: 0000-0001-6345-8711

Behaviour Design Lab - eHealth Center. Universitat Oberta de Catalunya

Autor corresponsal: Luis Ortiz-Muñoz

Correo: leortizmunoz@uc.cl

Dirección postal: Centro Evidencia UC. Pontificia Universidad Católica de Chile. Diagonal Paraguay \#362. Santiago, Chile. 


\section{RESUMEN:}

INTRODUCCIÓN: A nivel mundial, se estima que 9,8\% de las mujeres consumen alcohol durante el embarazo. La investigación actual sugiere que la ingesta de alcohol durante el embarazo pone en riesgo al recién nacido de sufrir discapacidades cognitivas y del desarrollo graves de por vida. Las intervenciones de cambio de comportamiento pueden ayudar a las mujeres a reducir su consumo de alcohol durante el embarazo. OBJETIVO: El objetivo de este análisis crítico es comprobar la validez y aplicabilidad de los resultados con respecto a la efectividad de intervenciones de cambio de comportamiento para reducir el consumo de alcohol en mujeres embarazadas, y responder a la interrogante: ¿Son efectivas las intervenciones de cambio de comportamiento en la reducción del consumo de alcohol en mujeres embarazadas?. MÉTODOS: Se realizó una búsqueda de evidencia proveniente de revisiones sistemáticas, sobre Intervenciones de cambio de comportamiento para la reducción del consumo de alcohol durante el embarazo. Se especificó para cada revisión los componentes PICO de la pregunta y, del listado de revisiones relevantes, se seleccionó la que pudiese responder mejor a la pregunta. RESULTADOS: Identificamos seis revisiones sistemáticas y dos revisiones panorámicas. Se analizó críticamente el artículo "Identifying effective behavior change techniques for alcohol and illicit substance use during pregnancy: a systematic review." CONCLUSIÓN: Existe evidencia para decir que algunas técnicas de cambio de comportamiento podrían ser útiles para lograr la reducción del consumo de alcohol durante el embarazo. Sin embargo, estos resultados se deben tomar con precaución dado algunas limitaciones metodológicas en la evidencia disponible.

\section{Artículo analizado:}

Fergie L. Identifying effective behavior change techniques for alcohol and illicit substance use during pregnancy: a systematic review. Annals of Behavioral Medicine. 2019 Aug;53(8):769-81

Palabras claves: Behavior change techniques, pregnancy, alcohol consumption.

\section{Ideas clave:}

- Existe evidencia para decir que algunas técnicas de cambio de comportamiento podrían ser útiles para lograr la reducción del consumo de alcohol durante el embarazo.

- Existen cinco técnicas de cambio de comportamiento que presentan una efectividad del $100 \%$, en la abstención y/o reducción del consumo de alcohol durante el embarazo.

- Por otro lado, estos resultados se deben tomar con precaución dado algunas limitaciones metodológicas en la evidencia disponible: Riesgo de sesgo, imprecisión y el hecho de que todos los estudios evaluaron la efectividad de las intervenciones con herramientas autoaplicadas. 


\section{Introducción:}

Muchos de los problemas de salud que enfrenta la población mundial no son de naturaleza médica ni científica; sino de comportamiento. Comprender e incentivar el uso e integración de las ciencias del comportamiento es importante para la salud pública [1]. A diario cada individuo toma una serie de decisiones que tienen un impacto en la propia salud y la salud de otros, siendo probable que algunos comportamientos sean más dañinos como el consumo de alcohol durante el embarazo.

Los comportamientos de riesgo para la salud son los principales contribuyentes a la morbilidad, la mortalidad prematura asociada con enfermedades crónicas y el aumento de los costos de salud. El consumo excesivo de alcohol es uno de los factores que ocasionan más problemas sociosanitarios [2], aumentando el riesgo de enfermedades crónicas, lesiones no intencionales, violencia y daño durante el embarazo.

Los comportamientos son impulsados por múltiples factores, como la motivación, la emoción, el entorno social y físico,incluyendo en este último los factores socioeconómicos y circunstancias familiares. El consumo de alcohol durante el embarazo es intuitivamente arriesgado[3], y sólo la ciencia puede establecer la magnitud real del riesgo. La percepción del riesgo es un constructo subjetivo que puede ser distorsionado por sesgos y heurísticas [4][5], obstaculizando los planes de promoción y prevención en salud. Así mismo, muchas intervenciones dirigidas a cambio de comportamiento tienen como objetivo aumentar la conciencia sobre los riesgos para la salud a través de estrategias basadas en el suministro de información a través de acciones comunicativas. Pero a menudo el problema principal no es una simple falta de información o conciencia [6], sino que es necesario incorporar técnicas basadas en la evidencia y

fundamentadas en teorías científicas. De esta manera, se puede responder a las necesidades de la sociedad, romper el status quo y mejorar la efectividad de algunas intervenciones para el cambio de comportamiento [7][8].

El uso de alcohol durante el embarazo puede causar daños en el sistema nervioso central del feto que se traducirán en manifestaciones físicas, mentales y conductuales, así como problemas de aprendizaje que pueden persistir a lo largo de la vida y que constituyen los que se conocen como trastornos del espectro alcohólico fetal (TEAF) [1] . Los TEAF se asocian a diferentes complicaciones, entre las que destacan las malformaciones congénitas, anomalías cromosómicas, retraso del crecimiento prenatal y postnatal, discapacidad intelectual, trastornos del comportamiento, alteraciones visuales y auditivas, malformaciones cardíacas y problemas genitourinarios. Este reto no es menor, puesto que se estima que a nivel mundial se estima que un $9,8 \%$ de las mujeres consumen alcohol durante el embarazo [2] . La investigación actual sugiere que la ingesta de siete o más bebidas estándar (una bebida estándar =13,6 gramos de alcohol absoluto) por semana, durante el embarazo, pone en riesgo al recién nacido, aunque no existen pruebas sólidas de la ausencia de efectos perjudiciales sobre el feto cuando se ingieren cantidades menores de alcohol [3]. Por lo tanto, son necesarias intervenciones psicológicas y educativas que puedan ayudar a las mujeres a abandonar o reducir su consumo de alcohol durante el embarazo [9]. 


\section{A. Pregunta clínica:}

¿¿Qué técnicas de cambio de comportamiento son las más efectivas en las intervenciones encaminadas a la abstención o reducción del consumo de alcohol en mujeres embarazadas??

\section{B. Metodos:}

\section{Criterios de inclusión:}

Se incluyeron las revisiones sistemáticas amplias, exactas o precisas que tratan sobre intervenciones de cambio de comportamiento para la reducción del consumo de alcohol durante el embarazo.

Estrategia de búsqueda:

Las bases de datos utilizadas fueron: Cochrane database of systematic reviews (CDSR); Database of Abstracts of Reviews of Effectiveness (DARE); HTA Database; PubMed; LILACS; CINAHL; PsycINFO; EMBASE; EPPI-Centre Evidence Library; WHO institutional Repository for information Sharing; NICE public health guidelines and systematic reviews; The JBI Database of Systematic Reviews and Implementation Reports, entre otras, a través de una búsqueda avanzada en la plataforma Epistemonikos. No se aplicaron restricciones en base al idioma o estado de publicación. La estrategia de búsqueda se elaboró en torno a los componentes de la pregunta, con identificación de términos relevantes en otras revisiones sistemáticas y/o thesaurus de bases de datos (detalles en anexo 1).

\section{Evaluación crítica:}

La evaluación crítica comienza con la valoración de la confianza general en los resultados de la revisión, con la valoración únicamente de los dominios críticos en la herramienta AMSTAR-2 [10], a lo que denominamos "AMSTAR 2 Abreviado", utilizado en proyectos anteriores para evaluar la confianza general en los resultados de las revisiones[11] (detalles en anexo 2). Luego se realiza una evaluación de la confianza en las estimaciones del efecto. Esta parte del proceso de análisis crítico es realizada con el segundo juicio de análisis presentado en artículo publicado por Murad et al. [12]. 


\section{Resultados:}

\section{Resumen de la evidencia identificada:}

Se buscaron revisiones sistemáticas que analizan estudios sobre la efectividad de las intervenciones de cambio de comportamiento en la abstención o reducción del consumo de alcohol en mujeres embarazadas. Se identificaron 6 revisiones sistemáticas y 2 revisiones panorámicas.

Tabla 1: Resumen de la evidencia identificada

\begin{tabular}{|l|l|}
\hline Revisiones sistemáticas & $6[9,13-17]$ \\
\hline Revisiones panorámicas & $2[18-19]$ \\
\hline
\end{tabular}

\section{Selección de la evidencia:}

Se realizó un análisis de las revisiones sistemáticas encontradas y se identificó cada componente PICO de la pregunta que abordaban. Para la evaluación crítica, se seleccionó una revisión sistemática ponderando los siguientes puntos: fecha de publicación, número de estudios incluidos, si respondía a la pregunta de manera exacta, específica o amplia y, finalmente, si era una revisión publicada por la Colaboración Cochrane (detalles en anexo 3). La revisión seleccionada fue: Fergie L. Identifying effective behavior change techniques for alcohol and illicit substance use during pregnancy: a systematic review. Annals of Behavioral Medicine. 2019 Aug;53(8):769-81.

\section{Evaluación crítica:}

Valoración de la confianza general en los resultados de la revisión:

Si bien no existen limitaciones en los dominios críticos, los ítems 1 y 11 presentan algunos problemas [Item 1: Sin protocolo propio, pero se basan en protocolos de revisiones sistemáticas Cochrane previas que abordan la misma pregunta. Ítem 11: No se realizan metanálisis]. Consideramos que la confianza en la revisión sistemática es calificada como MEDIA, lo que implica que: La revisión tiene debilidades, pero no hay defectos críticos, pudiendo proporcionar un resumen preciso de los resultados de los estudios disponibles (detalles en anexo 2).

\section{Evaluación de la confianza en las estimaciones del efecto:}

En cuanto a la confianza en la estimación de los efectos, vemos que el riesgo de sesgo es importante. Si bien los resultados son consistentes entre los estudios, estos son imprecisos. La evidencia que responde a la pregunta de interés es directa y se puede presumir bajo o nulo sesgo de publicación. No se identifican en los estudios incluidos en la revisión, razones para aumentar la calificación de confianza (detalles en anexo 4). 


\section{E. Síntesis de evidencia y traducción del conocimiento:}

Existe evidencia para decir que algunas técnicas de cambio de comportamiento podrían ser útiles para lograr la reducción del consumo de alcohol durante el embarazo. La efectividad de las intervenciones de cambio de comportamiento para la reducción del consumo de alcohol en mujeres embarazadas, presentaron diferentes porcentajes de efectividad. La siguiente tabla presenta las técnicas de comportamiento, su codificación según la taxonomía BCTTV1 [20] y Taxonomía para cambio de comportamiento y consumo de alcohol [21], porcentaje de efectividad - logro del objetivo (calculado por los autores de la revisión sistemática analizada [13]) de abstención o reducción del consumo de alcohol e ideas claves para la implementación:

\begin{tabular}{|c|c|c|c|}
\hline $\begin{array}{l}\text { Técnicas de cambio } \\
\text { de comportamiento }\end{array}$ & Taxonomia & $\begin{array}{l}\text { Porcentaje de } \\
\text { efectividad }\end{array}$ & $\begin{array}{l}\text { Ideas clave como apoyo a la implementación por parte del } \\
\text { prestador de salud }\end{array}$ \\
\hline $\begin{array}{l}\text { Planificación de } \\
\text { acciones }\end{array}$ & $\begin{array}{c}1.4 \\
\text { (BCTTV1) }\end{array}$ & $100 \%$ & $\begin{array}{l}\text { Definición: Planificación rápida y detallada del desempeño del } \\
\text { comportamiento (debe incluir al menos una de contexto, } \\
\text { frecuencia, duración e intensidad). El contexto puede ser ambiental } \\
\text { (físico o social) o interno (físico, emocional o cognitivo) (incluye } \\
\text { 'Intenciones de implementación ' ) } \\
\text { Ejemplo de acción: Fomente un plan para llevar sus propias bebidas } \\
\text { al salir socialmente los fines de semana. }\end{array}$ \\
\hline $\begin{array}{l}\text { Contrato de } \\
\text { comportamiento }\end{array}$ & $\begin{array}{c}1.8 \\
(\mathrm{BCTTV} 1)\end{array}$ & $100 \%$ & $\begin{array}{l}\text { Definición: Cree una especificación escrita del comportamiento a } \\
\text { realizar, acordado por la persona, y atestiguado por otra. } \\
\text { Ejemplo de acción: Firma un contrato con la persona, por ejemplo } \\
\text { especificando no beber alcohol durante una semana. }\end{array}$ \\
\hline Indicaciones / señales & $\begin{array}{c}7.1 \\
\text { (BCTTV1) }\end{array}$ & $100 \%$ & $\begin{array}{l}\text { Definición: Introducir o definir estímulos sociales o ambientales con } \\
\text { el fin de indicar o señalar el comportamiento. El aviso o la señal } \\
\text { normalmente ocurriría en la hora o lugar de ejecución. } \\
\text { Ejemplo de acción: Pon una pegatina en el refrigerador para } \\
\text { recordarle a la gente que se tome opciones de bebidas no } \\
\text { alcohólicas. }\end{array}$ \\
\hline $\begin{array}{l}\text { Dialogo consigo } \\
\text { mismo }\end{array}$ & $\begin{array}{c}15.4 \\
(\mathrm{BCTTV} 1)\end{array}$ & $100 \%$ & $\begin{array}{l}\text { Definición: Estimular el diálogo interno positivo (en voz alta o en } \\
\text { silencio) antes y durante el comportamiento. } \\
\text { Ejemplo de acción: Indicar a la persona que se diga a ellos mismos } \\
\text { que un estar sin beber es saludable para ella y el bebé. }\end{array}$ \\
\hline $\begin{array}{l}\text { Ofrecer / dirigir hacia } \\
\text { materiales escritos } \\
\text { apropiados }\end{array}$ & $\begin{array}{l}\text { BCT.30 } \\
\text { (taxonomía } \\
\text { del alcohol) }\end{array}$ & $100 \%$ & $\begin{array}{l}\text { Entregue o recomiende materiales escritos que puedan ser } \\
\text { apropiados para entregar información respecto de beneficios de la } \\
\text { abstención y/o reducción del consumo de alcohol durante el } \\
\text { embarazo. }\end{array}$ \\
\hline
\end{tabular}




\begin{tabular}{|c|c|c|c|}
\hline $\begin{array}{l}\text { Técnicas de cambio } \\
\text { de comportamiento }\end{array}$ & Taxonomia & $\begin{array}{l}\text { Porcentaje de } \\
\text { efectividad }\end{array}$ & Apoyo a la implementación por parte del prestador de salud \\
\hline $\begin{array}{l}\text { Resolución de } \\
\text { problemas }\end{array}$ & $\begin{array}{c}1.2 \\
(\mathrm{BCTT} \operatorname{T} 1)\end{array}$ & $67 \%$ & $\begin{array}{l}\text { Definición: Analizar, o incitar a la persona a analizar, factores que } \\
\text { influyen en el comportamiento y generar o seleccionar estrategias } \\
\text { que incluyan superar barreras y / o aumentar facilitadores. } \\
\text { Ejemplo de acción: Identificar desencadenantes específicos ( } \mathrm{p} \text {. Ej. } \\
\text { estar en un pub, sentirse ansioso) que generan la urgencia / deseo / } \\
\text { necesidad de beber y desarrollar estrategias para evitar } \\
\text { desencadenantes ambientales o para manejar las emociones } \\
\text { negativas, como la ansiedad, que le motivan a beber. }\end{array}$ \\
\hline $\begin{array}{l}\text { Retroalimentación } \\
\text { del } \\
\text { comportamiento }\end{array}$ & $\begin{array}{c}2.2 \\
(\mathrm{BCTT} \operatorname{T} 1)\end{array}$ & $67 \%$ & $\begin{array}{l}\text { Definición: Supervisar y proporcionar información o } \\
\text { retroalimentación evaluativa sobre el desempeño de el } \\
\text { comportamiento (por ejemplo, forma, frecuencia, duración, } \\
\text { intensidad) } \\
\text { Ejemplo de acción: Informar a la persona de cuántas episodios de } \\
\text { consumo de alcohol tuvo en una semana. (por ejemplo, basado en } \\
\text { un cuestionario de consumo de alcohol). }\end{array}$ \\
\hline $\begin{array}{l}\text { Apoyo social (no } \\
\text { especificado) }\end{array}$ & $\begin{array}{c}3.1 \\
(\mathrm{BCTT} \operatorname{l} 1)\end{array}$ & $67 \%$ & $\begin{array}{l}\text { Definición: Asesorar, organizar o proporcionar servicios sociales de } \\
\text { apoyo (por ejemplo, de amigos, familiares, colegas, 'amigos' o } \\
\text { personal). } \\
\text { Ejemplo de acción: Aconseje a la persona que llame a un 'amigo' } \\
\text { cuando experimente deseos de beber alcohol. }\end{array}$ \\
\hline $\begin{array}{l}\text { Información sobre } \\
\text { consecuencias de } \\
\text { salud. }\end{array}$ & $\begin{array}{c}5.1 \\
(\mathrm{BCTT} \operatorname{1} 1)\end{array}$ & $67 \%$ & $\begin{array}{l}\text { Definición: Proporcionar información (por ejemplo, escrita, verbal, } \\
\text { visual) sobre las consecuencias para la salud de realizar el } \\
\text { comportamiento. } \\
\text { Ejemplo de acción: Explique las consecuencias en la salud que } \\
\text { puede tener la madre y/o el recién nacido producto del alcohol. }\end{array}$ \\
\hline $\begin{array}{l}\text { Sustitución del } \\
\text { comportamiento }\end{array}$ & $\begin{array}{c}8.2 \\
(\mathrm{BCTT} \operatorname{T} 1)\end{array}$ & $67 \%$ & $\begin{array}{l}\text { Definición: Sustitución inmediata del comportamiento no deseado } \\
\text { por uno neutral o deseado. } \\
\text { Ejemplo de acción: Sugerir que la persona vaya por un paseo/ tome } \\
\text { un libro/entre otros en lugar de beber. }\end{array}$ \\
\hline $\begin{array}{l}\text { Evaluar la } \\
\text { preparación actual y } \\
\text { la capacidad para } \\
\text { reducir el consumo } \\
\text { excesivo de alcohol. }\end{array}$ & $\begin{array}{l}\text { BCT.29 } \\
\text { (taxonomía } \\
\text { del alcohol) }\end{array}$ & $67 \%$ & $\begin{array}{l}\text { Evalúe junto a la persona como es su preparación y capacidad } \\
\text { actual de reducir (o abstenerse) el consumo de alcohol. }\end{array}$ \\
\hline
\end{tabular}




\begin{tabular}{|l|c|c|c|}
\hline $\begin{array}{l}\text { Técnicas de cambio } \\
\text { de comportamiento }\end{array}$ & Taxonomia & $\begin{array}{l}\text { Porcentaje de } \\
\text { efectividad }\end{array}$ & Apoyo a la implementación por parte del prestador de salud \\
\hline $\begin{array}{l}\text { Fijar metas } \\
\text { (comportamiento). }\end{array}$ & $\begin{array}{c}1.1 \\
\text { (BCTTV1) }\end{array}$ & $50 \%$ & $\begin{array}{l}\text { Definición: Establecer o acordar un objetivo definido en términos } \\
\text { del comportamiento a lograr. } \\
\text { Ejemplo de acción: Acuerde un objetivo diario de abstención (por } \\
\text { ejemplo, 1 mes) con la persona y llegar a un acuerdo sobre la meta. }\end{array}$ \\
\hline $\begin{array}{l}\text { Adaptación de las } \\
\text { interacciones de } \\
\text { manera apropiada. }\end{array}$ & $\begin{array}{c}\text { BCT35 } \\
\text { (Taxonomía } \\
\text { del alcohol) }\end{array}$ & $50 \%$ & Sin definición oficial por parte de los creadores de la taxonomía. \\
\hline
\end{tabular}

\section{E. Ámbitos:}

Debido a la variación internacional y temporal en las recomendaciones para beber de forma segura durante el embarazo, los resultados de los estudios sobre el alcohol variaron a lo largo del tiempo y entre países. En consecuencia, utilizamos los resultados de abstinencia o reducción del consumo de alcohol.

\begin{tabular}{|l|l|}
\hline Ambito & Observaciones \\
\hline $\begin{array}{l}\text { Escocia, Irlanda, EEUU., } \\
\text { Canadá, Australia y Nueva } \\
\text { Zelanda }\end{array}$ & $\begin{array}{l}\text { Las pautas abogan sistemáticamente por la abstinencia únicamente, sin } \\
\text { que se considere que ninguna cantidad de alcohol sea segura durante el } \\
\text { embarazo. }\end{array}$ \\
\hline Inglaterra & $\begin{array}{l}\text { Las pautas ahora abogan por la abstinencia, antes de 2016, las } \\
\text { recomendaciones eran tales que la abstinencia era preferible, pero si no } \\
\text { se podía lograr, entonces uno o dos tragos, una o dos veces por semana } \\
\text { sin emborracharse, era el límite superior recomendado. para evitar } \\
\text { riesgos fetales. }\end{array}$ \\
\hline
\end{tabular}

\section{F. Limitaciones:}

La evidencia disponible presenta varias limitaciones. Por lo general, las intervenciones de cambio de comportamiento contienen varias técnicas de cambio de comportamiento y se piensa que interactúan entre sí. En la revisión analizada, las técnicas de cambio de comportamiento se evaluaron de manera independiente, por lo que se podría dudar si los resultados se deben o no a esas interacciones. Además, todos los estudios primarios incluidos midieron los resultados de sus intervenciones con reporte autoinformado, sin validación de antecedentes bioquímicos, por lo tanto, los resultados a favor de la intervención podrían ser explicados por un sesgo en las respuestas de los reportes autoinformados.

Este manuscrito es parte de un proyecto de síntesis de evidencia liderado por Centro Evidencia UC y Postgrado NYD UC [22] de la Pontificia Universidad Católica de Chile, en colaboración con Behaviour Design Lab - eHealth Center. Universitat Oberta de Catalunya. 


\section{Referencias:}

1. OMS. (2020). Behavioural Insights.

https://www.who.int/our-work/science-division/behavioural-insights

2. CDC. (2021a, mayo 21). Basics about FASDs. Centers for Disease Control and Prevention. https://www.cdc.gov/ncbddd/fasd/facts.html

3. CDC. (2021b, mayo 24). Alcohol Use in Pregnancy. Centers for Disease Control and Prevention. https://www.cdc.gov/ncbddd/fasd/alcohol-use.html

4. Slovic, P. (1987). Perception of risk. Science, 236(4799), 280-285. https://doi.org/10.1126/science.3563507

5. Tversky, A., \& Kahneman, D. (1974). Judgment under Uncertainty: Heuristics and Biases. Science, 185(4157), 1124-1131. https://doi.org/10.1126/science.185.4157.1124

6. Steinberg, L. (2015). How to Improve the Health of American Adolescents. Perspectives on Psychological Science: A Journal of the Association for Psychological Science, 10(6), 711-715. https://doi.org/10.1177/1745691615598510

7. Michie, S., van Stralen, M. M., \& West, R. (2011). The behaviour change wheel: A new method for characterising and designing behaviour change interventions. Implementation Science : IS, 6 , 42. https://doi.org/10.1186/1748-5908-6-42

8. Michie, S., Wood, C. E., Johnston, M., Abraham, C., Francis, J. J., Hardeman, W., Michie, S., Wood, C. E., Johnston, M., Abraham, C., Francis, J. J., \& Hardeman, W. (2015). Técnicas de cambio de comportamiento: El desarrollo y evaluación de un método taxonómico para informar y describir intervenciones de cambio de comportamiento (un conjunto de cinco estudios que incluyen métodos de consenso, ensayos controlados aleatorios y análisis de datos cualitativos). Biblioteca de revistas de NIHR.

9. Stade BC, Bailey C, Dzendoletas D, Sgro M, Dowswell T, Bennett D. Psychological and/or educational interventions for reducing alcohol consumption in pregnant women and women planning pregnancy. Cochrane Database of Systematic Reviews. 2009(2).

10. Ciapponi A. AMSTAR-2: herramienta de evaluación crítica de revisiones sistemáticas de estudios de intervenciones de salud. Evidencia, actualización en la práctica ambulatoria. 2018 Apr 1;21(1).

11. Ortiz-Muñoz LE, Pantoja T, Morel M. Resumen SUPPORT de una revisión sistemática : Cuarentena sola o en combinación con otras medidas de salud pública para controlar el COVID-19: una revisión rápida. Satellite Cochrane EPOC-CHILE 2020. doi:10.31219/osf.io/kx6pn.

12. Murad MH, Montori VM, loannidis JP, Jaeschke R, Devereaux PJ, Prasad K, Neumann I, Carrasco-Labra A, Agoritsas T, Hatala R, Meade MO. How to read a systematic review and meta-analysis and apply the results to patient care: users' guides to the medical literature. Jama. 2014 Jul 9;312(2):171-9.

13. Fergie L, Campbell KA, Coleman-Haynes T, Ussher M, Cooper S, Coleman T. Identifying effective behavior change techniques for alcohol and illicit substance use during pregnancy: a systematic review. Annals of Behavioral Medicine. 2019 Aug;53(8):769-81.

14. de Paula Gebara CF, de Castro Bhona FM, Ronzani TM, Lourenço LM, Noto AR. Brief intervention and decrease of alcohol consumption among women: a systematic review. Substance abuse treatment, prevention, and policy. 2013 Dec;8(1):1-8. 
15. Mosd $\varnothing \mid$ A, Lidal IB, Straumann GH, Vist GE. Targeted mass media interventions promoting healthy behaviours to reduce risk of non-communicable diseases in adult, ethnic minorities. Cochrane Database of Systematic Reviews. 2017(2).

16. Gilinsky A, Swanson V, Power K. Interventions delivered during antenatal care to reduce alcohol consumption during pregnancy: A systematic review. Addiction Research \& Theory. 2011 Jun 1;19(3):235-50.

17. Whitworth $M$, Dowswell $T$. Routine pre-pregnancy health promotion for improving pregnancy outcomes. Cochrane Database of Systematic Reviews. 2009(4).

18. Heslehurst N, Hayes L, Jones D, Newham J, Olajide J, McLeman L, McParlin C, De Brún C, Azevedo L. The effectiveness of smoking cessation, alcohol reduction, diet and physical activity interventions in changing behaviours during pregnancy: A systematic review of systematic reviews. PloS one. 2020 May 29;15(5):e0232774.

19. Hayes L, McParlin C, Azevedo LB, Jones D, Newham J, Olajide J, McCleman L, Heslehurst N. The Effectiveness of Smoking Cessation, Alcohol Reduction, Diet and Physical Activity Interventions in Improving Maternal and Infant Health Outcomes: A Systematic Review of Meta-Analyses. Nutrients. 2021 Mar;13(3):1036.

20. Michie S, Richardson M, Johnston M, Abraham C, Francis J, Hardeman W, Eccles MP, Cane J, Wood CE. The behavior change technique taxonomy (v1) of 93 hierarchically clustered techniques: building an international consensus for the reporting of behavior change interventions. Annals of behavioral medicine. 2013 Aug 1;46(1):81-95.

21. Michie S, Whittington C, Hamoudi Z, Zarnani F, Tober G, West R. Identification of behaviour change techniques to reduce excessive alcohol consumption. Addiction. 2012 Aug;107(8):1431-40.

22. Ortiz-Muñoz LE, Fredes C, Rojas-Sobarzo L, Daza AP. Proyecto de síntesis de evidencia en nutrición y dietética. Centro Evidencia UC - Postgrado NYD UC 2021. https://osf.io/pghzv/ 
Anexo 1: Términos utilizados para la estrategia de búsqueda:

\section{Cambio de comportamiento:}

1. behavioral choice theory

2. economic theory

3. behavioral economic theory

4. behaviour change interventions

5. behavioural change

6. behavior change

7. change interventions

8. intervention design

9. behavior change interventions

10. comprehensive messaging strategy

11. behavior-change interventions

12. long-term behaviour change

13. behavioural interventions

14. health behaviour change

15. behavioral change techniques

16. nudge

\section{Embarazo:}

1. pregnan*

2. maternal

3. gestation

\section{Consumo de alcohol:}

1. Alcohol AND ( intake OR drinking OR excessive OR consumption) 
Anexo 2: Valoración de la confianza general en los resultados de la revisión

\section{Valoración de la confianza general en los resultados de la revisión}

Artículo analizado: Identifying effective behavior change techniques for alcohol and illicit substance use during pregnancy: a systematic review. Annals of Behavioral Medicine. 2019 Aug;53(8):769-81

\begin{tabular}{|l|l|c|}
\hline Item 1 & Protocolo registrado antes de la revisión & Sí $^{*}$ \\
\hline Item 4 & Adecuada búsqueda en la literatura & Sí \\
\hline Item 7 & Justificación de los estudios excluidos & Sí Sí \\
\hline Item 9 & Riesgo de sesgo de los estudios individuales incluidos & Si** \\
\hline Item 11 & Métodos meta-analíticos apropiados & Sí \\
\hline Item 13 & Consideración del riesgo de sesgo en la interpretación de los resultados de la revisión & Sí \\
\hline Item 15 & Evaluación de la presencia y el impacto probable del sesgo de publicación & \\
\hline
\end{tabular}

Limitaciones: Si bien hay no hay limitaciones en los dominios críticos, los ítem 1 y 11 presentan algunos problemas [*Sin protocolo propio, pero se basan en protocolos de revisiones sistemáticas Cochrane previas, que abordan la misma pregunta. ${ }^{* *}$ No se realizan metanálisis ], consideramos que la confianza en la revisión sistemática es calificada como MEDIA, lo que implica que: La revisión tiene debilidades, pero no hay defectos críticos, pudiendo proporcionar un resumen preciso de los resultados de los estudios disponibles.

Tabla 2: AMSTAR abreviado donde se evalúan los dominios críticos de la herramienta AMSTAR 2. Elaboración propia.

\begin{tabular}{|l|l|}
\hline Confianza & Justificación \\
\hline Alta & $\begin{array}{l}\text { Ninguna debilidad crítica y hasta una no crítica: La RS proporciona un resumen exacto y completo de } \\
\text { los resultados de los estudios disponibles. }\end{array}$ \\
\hline Media & $\begin{array}{l}\text { Ninguna debilidad crítica y más de una no crítica: La RS tiene debilidades, pero no hay defectos } \\
\text { críticos, pudiendo proporcionar un resumen preciso de los resultados de los estudios disponibles. }\end{array}$ \\
\hline Baja & $\begin{array}{l}\text { Hasta una debilidad crítica, con o sin debilidades no críticas: La RS puede no proporcionar un resumen } \\
\text { exacto y completo de los estudios disponibles. }\end{array}$ \\
\hline Criticamente Baja & Más de una debilidad crítica, con o sin debilidades no críticas: La RS no es confiable. \\
\hline
\end{tabular}

Adaptado de Ciapponi A. AMSTAR-2: herramienta de evaluación crítica de revisiones sistemáticas de estudios de intervenciones de salud. Evidencia, actualización en la práctica ambulatoria. 2018 Apr 1;21(1). 
Anexo 3: Análisis de revisiones sistemáticas y otros manuscritos encontrados en la búsqueda.

\begin{tabular}{|c|c|c|c|c|c|c|c|c|}
\hline Ref & Año & $\begin{array}{l}\text { Estudios } \\
\text { incluidos }\end{array}$ & Población & Intervención & Comparación & Desenlace & Tipo de $\mathbf{R S}^{2}$ & $\begin{array}{l}\text { ¿RS } \\
\text { Cochrane? }\end{array}$ \\
\hline $13^{1}$ & 2019 & 9 & $\begin{array}{l}\text { Mujeres } \\
\text { embarazadas }\end{array}$ & $\begin{array}{l}\text { Intervenciones de } \\
\text { cambio de } \\
\text { comportamiento. }\end{array}$ & N/A & $\begin{array}{l}\text { Reducción } \\
\text { del consumo } \\
\text { de alcohol y } \\
\text { sustancias } \\
\text { ilícitas. }\end{array}$ & Amplia & No \\
\hline 9 & 2009 & 6 & $\begin{array}{l}\text { Mujeres } \\
\text { embarazadas y } \\
\text { mujeres que planean } \\
\text { embarazarse. }\end{array}$ & $\begin{array}{l}\text { Intervenciones } \\
\text { psicológicas y / o } \\
\text { educativas }\end{array}$ & N/A & $\begin{array}{l}\text { Reducción } \\
\text { del consumo } \\
\text { de alcohol. }\end{array}$ & Amplia & Sí \\
\hline 14 & 2013 & 36 & Mujeres & Intervención breve & N/A & $\begin{array}{l}\text { Reducción } \\
\text { del consumo } \\
\text { de alcohol. }\end{array}$ & Amplia & No \\
\hline 15 & 2017 & 7 & $\begin{array}{l}\text { Minorías étnicas } \\
\text { adultas. }\end{array}$ & $\begin{array}{l}\text { Cambios de } \\
\text { comportamiento. }\end{array}$ & N/A & $\begin{array}{l}\text { Reducir el } \\
\text { riesgo de } \\
\text { enfermedad } \\
\text { es no } \\
\text { transmisibles }\end{array}$ & Amplia & $\mathrm{Si}$ \\
\hline 16 & 2011 & \multicolumn{7}{|c|}{ Revisión sistemática sobre intervenciones ante natales } \\
\hline 17 & 2009 & \multicolumn{7}{|c|}{ Revisión sistemática sobre intervenciones ante natales } \\
\hline 18 & 2020 & \multicolumn{7}{|c|}{ Revisión panorámica } \\
\hline 19 & 2021 & \multicolumn{7}{|c|}{ Revisión panorámica } \\
\hline
\end{tabular}

${ }^{1}$ Revisión sistemática seleccionada para ser analizada por ser la más actual, presentar misma población e intervención de interés. Si bien presenta análisis del desenlace "consumo de sustancias ilícitas" evalúa de manera independiente las intervenciones para disminuir el consumo de alcohol.

${ }^{2}$ Tipo de revisiones sistemáticas posibles: Amplia, exacta, específica y no responde. 
Anexo 4: Evaluación de la confianza en las estimaciones del efecto.

\section{Evaluación de la confianza en las estimaciones del efecto}

Artículo analizado: Identifying effective behavior change techniques for alcohol and illicit substance use during pregnancy: a systematic review. Annals of Behavioral Medicine. 2019 Aug;53(8):769-81

\begin{tabular}{|l|l|}
\hline $\begin{array}{l}\text { ¿Qué tan serio es el riesgo de sesgo en el } \\
\text { Cuerpo de evidencia? }\end{array}$ & $\begin{array}{l}\text { Consideramos que el riesgo de sesgo es serio, dado que el riesgo de sesgo } \\
\text { en relación con el cegamiento de los participantes y el personal fue alto en } \\
\text { la mayoría de los ECA incluidos en los análisis finales. }\end{array}$ \\
\hline $\begin{array}{l}\text { ¿Los resultados de los distintos estudios son } \\
\text { consistentes? }\end{array}$ & $\begin{array}{l}\text { Consideramos que los resultados son consistentes, puesto que los } \\
\text { resultados no difieren tanto de un estudio a otro. (Sin cálculos de intervalos } \\
\text { de confianza formales en la revisión sistemática) }\end{array}$ \\
\hline ¿Qué tan precisos son los resultados? & $\begin{array}{l}\text { Consideramos los resultados como imprecisos, debido a que la muestra de } \\
\text { los estudios es pequeña. (Sin cálculos de estimación puntual de los estudios } \\
\text { combinados ni intervalos de confianza) }\end{array}$ \\
\hline $\begin{array}{l}\text { ¿Los estudios tienen pacientes, } \\
\text { las que vemos en la práctica clínica? }\end{array}$ & $\begin{array}{l}\text { Tanto la población, las intervenciones y los desenlaces en los estudios son } \\
\text { los mismos que la pregunta de interés de este resumen, la evidencia puede } \\
\text { ser considerada como directa. }\end{array}$ \\
\hline ¿Podría existir sesgo de reporte? & $\begin{array}{l}\text { Se presume bajo (o nulo) el sesgo de reporte, de hecho los autores de los } \\
\text { ensayos fueron contactados por los autores de las revisiones y estos } \\
\text { compartieron material adicional como por ejemplo libros de registros } \\
\text { entregados a los pacientes. }\end{array}$ \\
\hline $\begin{array}{l}\text { ¿Hay razones para aumentar la calificación de } \\
\text { confianza? }\end{array}$ & \begin{tabular}{l} 
No hay razones para aumentar la calificación de la confianza. \\
\hline
\end{tabular} \\
\hline
\end{tabular}

Tabla 3: Evaluación de la confianza en las estimaciones del efecto. Elaboración propia. 